\title{
Infrared Transmission Characteristics and Laser Tissue Interaction of Albino Pigskin Using Pulsed NIR Laser Light
}

\author{
Hisham Abdussamad Abbas and Gregory E. Triplett \\ Electrical Engineering, University of Missouri, Columbia, MO 65211, USA \\ Correspondence should be addressed to Gregory E. Triplett; triplettg@missouri.edu
}

Received 22 November 2012; Accepted 16 December 2012

Academic Editors: S. R. Restaino and X. Shu

Copyright (c) 2013 H. Abdussamad Abbas and G. E. Triplett. This is an open access article distributed under the Creative Commons Attribution License, which permits unrestricted use, distribution, and reproduction in any medium, provided the original work is properly cited.

\begin{abstract}
This work explores near infrared transmission through albino pigskin and determines controllable factors that influence transmission efficiency. Pigskin samples of varying thicknesses were irradiated using a $1440 \mathrm{~nm}$ near-infrared laser diode, where a photodetector was used to measure the transmitted power, and a two-dimensional real time surface temperature distribution was recorded using infrared thermography. Results demonstrate that this technique could potentially lead to a noninvasive approach for enhancing wound healing.
\end{abstract}

\section{Introduction}

In the last several decades, the study of electromagnetic irradiation and its influence on live tissue cells have been of great interest. Studies of laser-tissue interactions have expanded the application of photonics into the field of medicine [1]. The motivation for this particular study is to determine the parameters that influence transmission efficiency of laserirradiated tissue, which provides photonic energy to cells for biostimulation [2]. The human skin, which comprises of three different regions: the outer layer-epidermis, the middle layer-dermis, and the inner layer-hypodermis varies both in thickness and light transmission characteristics [3]. As such, there is skepticism that laser transmission through skin can serve as a feasible approach for wound healing. Numerous approaches to wound healing have been previously explored and most have not provided control over the entire healing process.

Photonic biostimulation, however, is an interesting approach to wound healing that has not been thoroughly explored. The key advantage of a photonics-based approach is reduced exposure of internal wounds to atmosphere, where the exposure to microorganisms is minimized. Ultraviolet, visible, or infrared wavelengths are candidates for photonic biostimulation; however, due to the absorption of ultraviolet and visible light on skin, these are not ideal for photonic biostimulation [4]. In contrast, infrared radiation has longer wavelength enabling it to penetrate further into the skin to reach targeted tissue and provide the needed excitation at the cellular level without cell damage [5]. When a molecule interacts with infrared light, there is a transfer of photon energy and momentum. The absorption characteristics of cellular organelles are the basis for wound healing via photonic stimulation [6].

A number of factors influence photon transmission: skin thickness, wavelength of incident beam, skin moisture content, dispersion, scattering, and absorption. The absorption can be approximated using Beer-Lamberts law:

$$
I=I_{o} e^{-u L},
$$

where $L$ is the skin thickness, $I$ is the transmitted intensity, $I_{o}$ is the incident intensity, and $u$ is the absorption coefficient (i.e., probability that a photon will be absorbed by the medium per unit length). The inverse of the absorption coefficient is the absorption length. The Beer-Lambert approximation is valid under certain conditions, that is, the light should be monochromatic and perfectly collimated and the medium should be pure and uniformly absorbing. However, biological tissue has many components called chromophores that absorb light nonuniformly based on its concentration and type. Hence, the Beer-Lambert law cannot be directly 


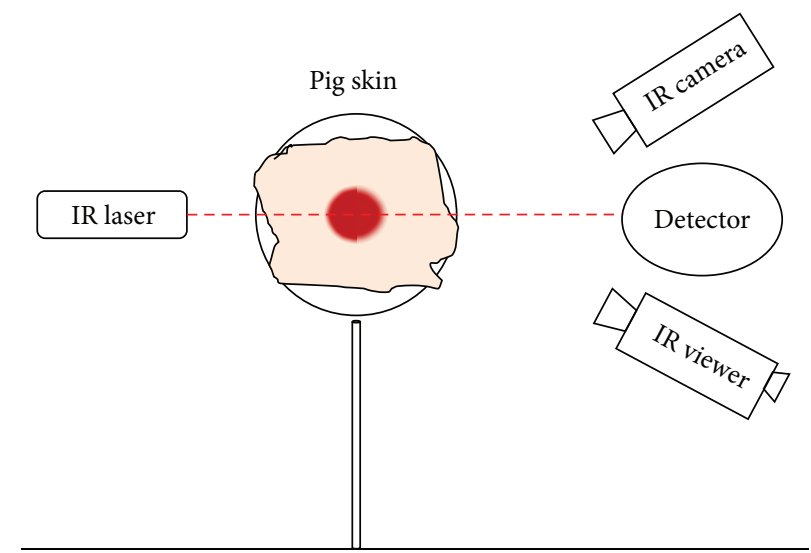

FIGURE 1: Experimental set-up during sample irradiation with infrared light.

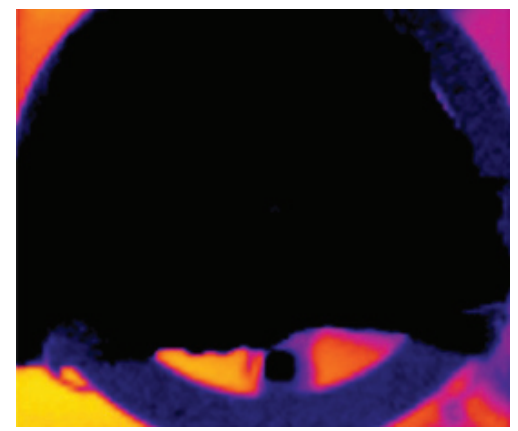

Before irradiation

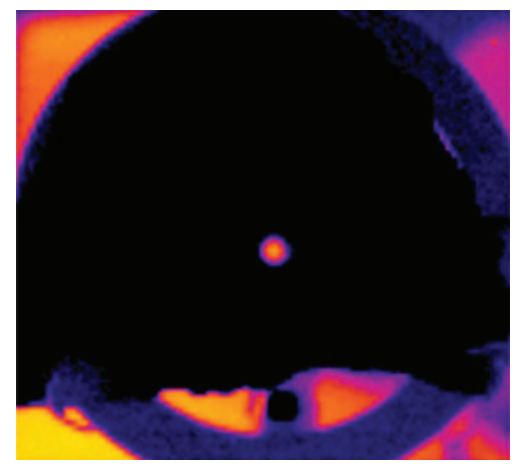

5 seconds of IR exposure

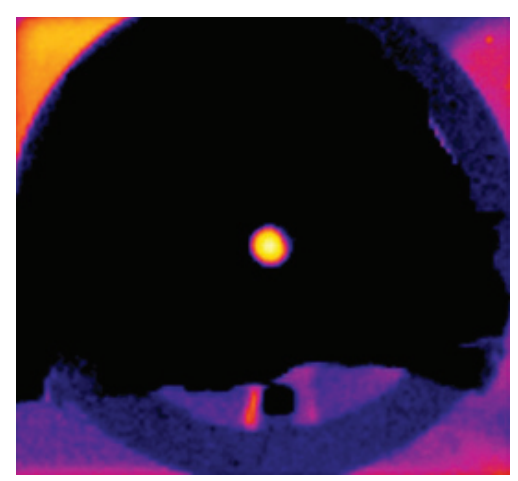

20 seconds of IR exposure

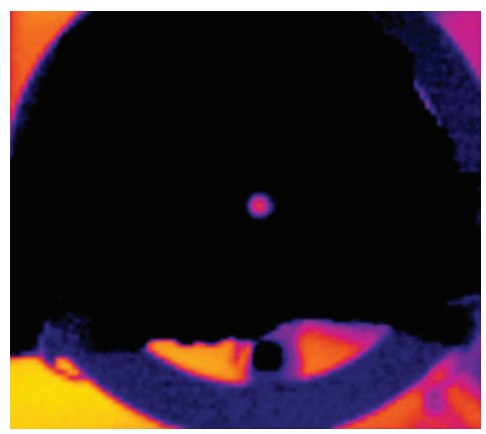

3 seconds of IR exposure

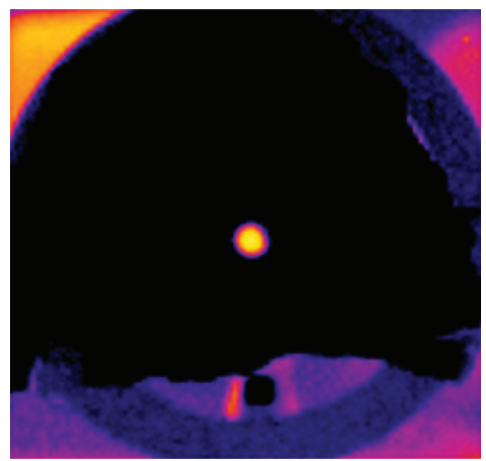

10 seconds of IR exposure

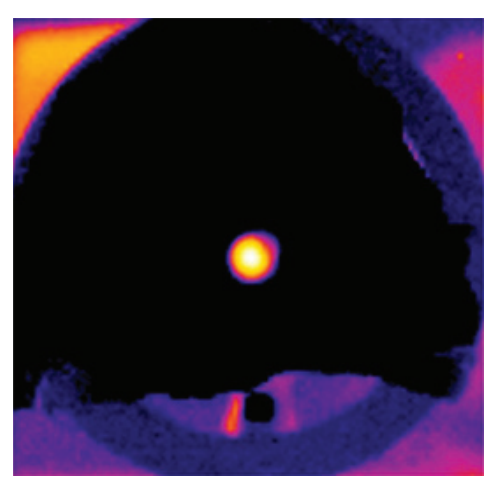

60 seconds of IR exposure

FIGURE 2: Infrared capture from onset of irradiation. 


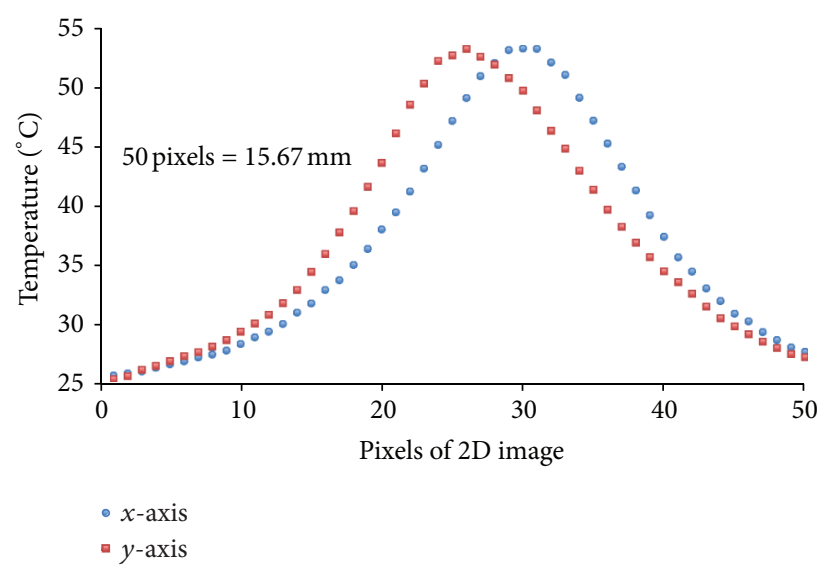

Figure 3: Temperature distributions along $x$ - and $y$-axes for $500-\mathrm{kHz}$ pulsed light at $1 \%$ duty cycle.

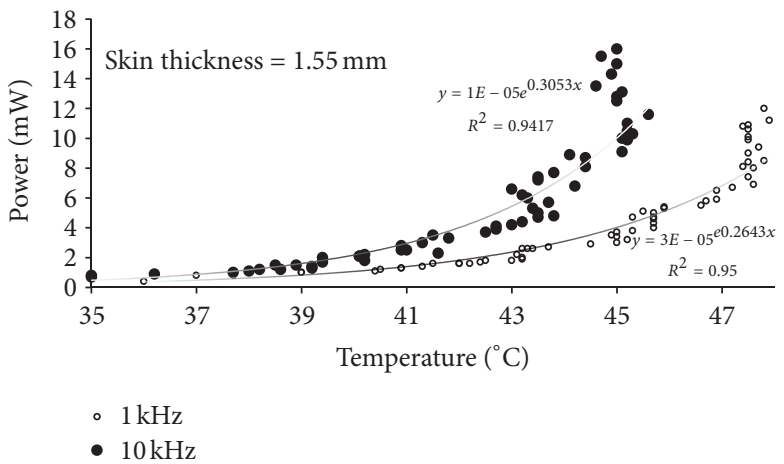

FIgURE 4: Transmission characteristics of $1-\mathrm{kHz}$ and $10-\mathrm{kHz}$ pulsed trains.

applied to biological tissue, as the total absorption coefficient is the sum of absorption coefficient of different individual chromophores. Nevertheless, Beer-Lambert law is still a good approximation and used for estimation purposes in this study.

\section{Experimental Method}

The test apparatus (Figure 1) was constructed to analyze the transmission, thermal, and temporal characteristics of irradiated pigskin. The transmitter side consisted of a laser diode, laser diode controller, and function generator. The infrared source used in this study is a $1440 \mathrm{~nm}$ single-mode fibercoupled Fabry Perot laser diode from QPhotonics (Model QFLD-1440-200S) with built-in thermistor and photodiode. The receiver side consisted of a germanium optical sensor from Thorlabs (Model S122B), which has a spectral range of $700-1800 \mathrm{~nm}$ and detection range of $35 \mathrm{nW}-35 \mathrm{~mW}$, and a thermal imaging camera from FLIR systems (Thermovision A20), that has a spectral range of $7.5-13 \mu \mathrm{m}$, sensitivity of $0.12^{\circ} \mathrm{C}$, accuracy of $\pm 2^{\circ} \mathrm{C}$, and temperature range of $-20^{\circ} \mathrm{C}$, to $250^{\circ} \mathrm{C}$.

The optical power and skin temperature were measured using the germanium sensor and infrared camera, respectively. Even though pigskin is $5-8$ times thicker than human skin, it is employed in this study because it is the closest in structure and transmission characteristics to human skin [7]. To ensure all skin samples are uniform and to preserve their natural condition, the hair follicles on the pigskin and underlying fat are removed and the processed skin sample is vacuum-sealed and refrigerated. The thickness of each sample was measured using a cross-section of the sample in a microscope.

The pulsed infrared light was provided at constant optical power and at a fixed distance from the skin sample. Pulsed irradiation was used primarily for controlling sample temperature. Each sample was irradiated at various positions along the skin surface to enable a comparative study. The power transmitted through the skin is detected using a sensor, and thermal transients along the surface are monitored using a thermal camera. The influence of pulsed infrared light is studied by varying the pulse frequency, duty cycle, and pulse width of the beam. The principal goal of the study is to maximize transmission efficiency of laser-irradiated skin while minimizing thermal losses.

When the sample is irradiated, a portion of photon energy is transferred to the surrounding skin demonstrating that thermal transfer governs the transmission characteristics. The transferred energy signifies the presence of dissipation and absorption losses, which must be minimized. Figure 2 shows infrared image capture in sequence of time from 


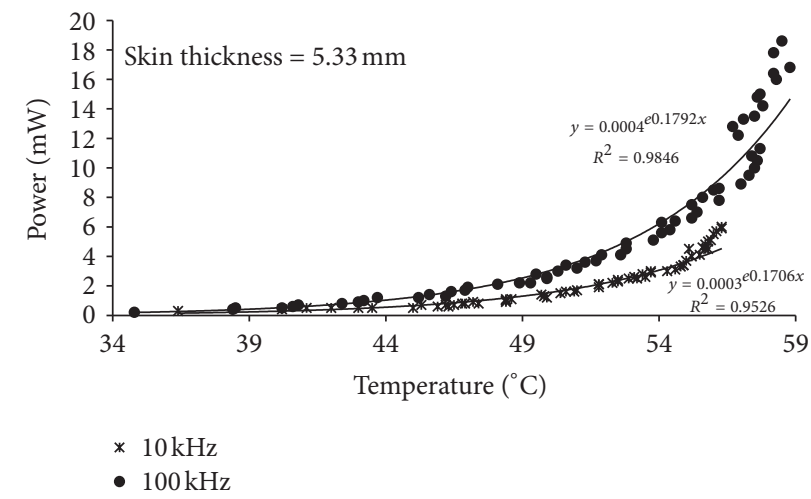

FIGURE 5: Transmission characteristics of $10-\mathrm{kHz}$ and $100-\mathrm{kHz}$ pulsed trains.

$\mathrm{O} / \mathrm{P}-2.2 \mathrm{~mW}$-spot width $=13.07 \mathrm{~mm}$

$10 \mathrm{kHz}$

$50-55^{\circ} \mathrm{C}$,

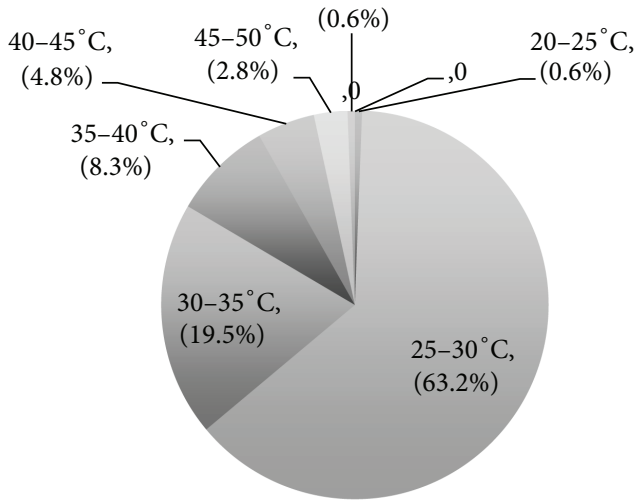

$\mathrm{O} / \mathrm{P}-4.5 \mathrm{~mW}$-spot width $=12.63 \mathrm{~mm}$

$100 \mathrm{kHz}$

$$
45-50^{\circ} \mathrm{C}
$$

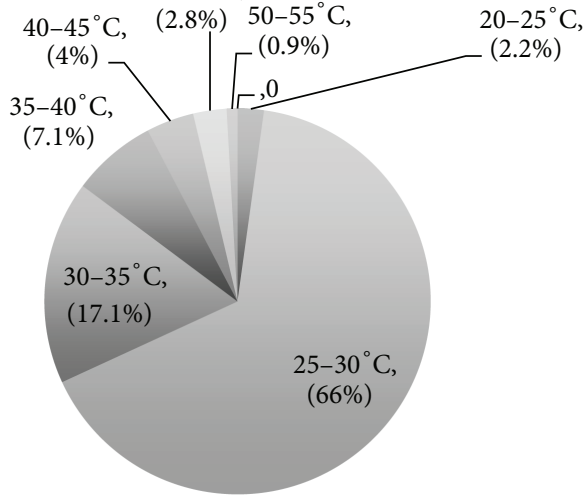

Figure 6: Pixel map of $10-\mathrm{kHz}$ and $100-\mathrm{kHz}$ pulse trains.

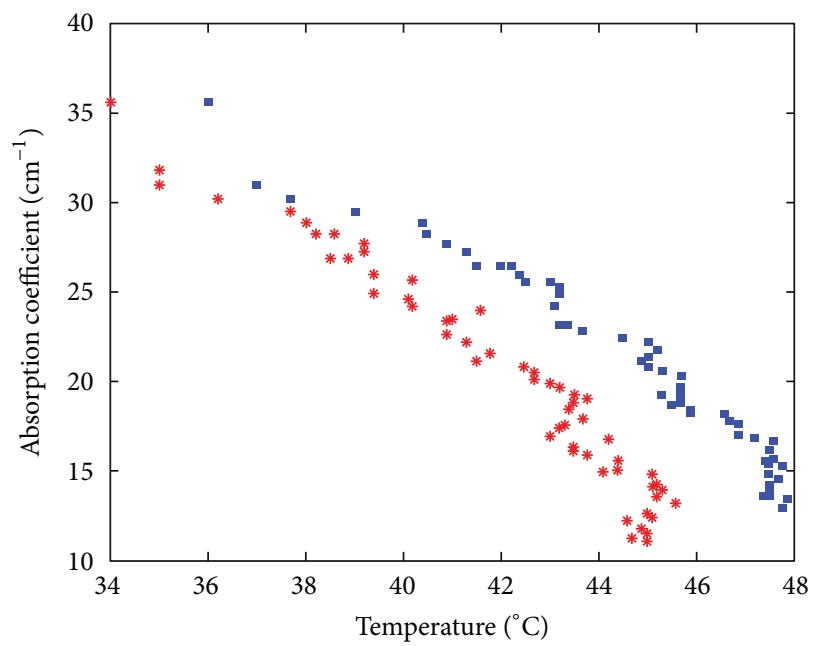

- $1 \mathrm{kHz}$

* $10 \mathrm{kHz}$

FIgURE 7: Absorption of 1-kHz and 10-kHz pulsed trains. 


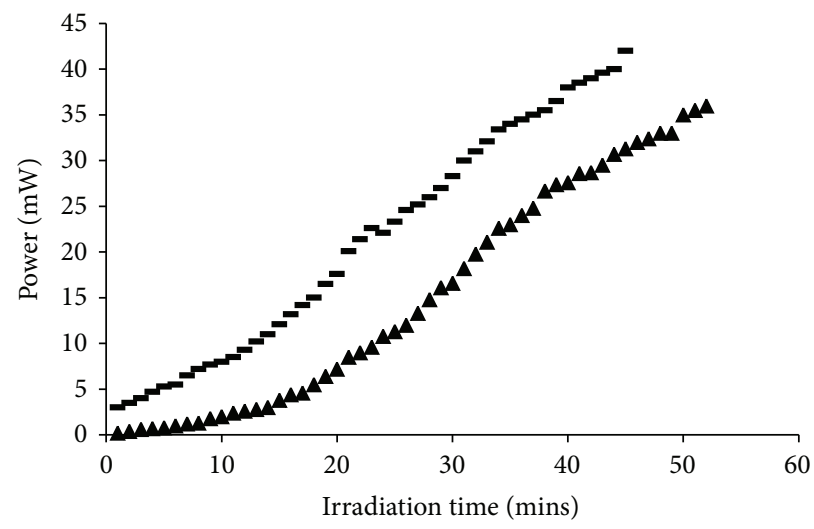

- $3 \%$ duty cycle

- $5 \%$ duty cycle

Figure 8: Temporal characteristics of 3\% and 5\% duty cycle.

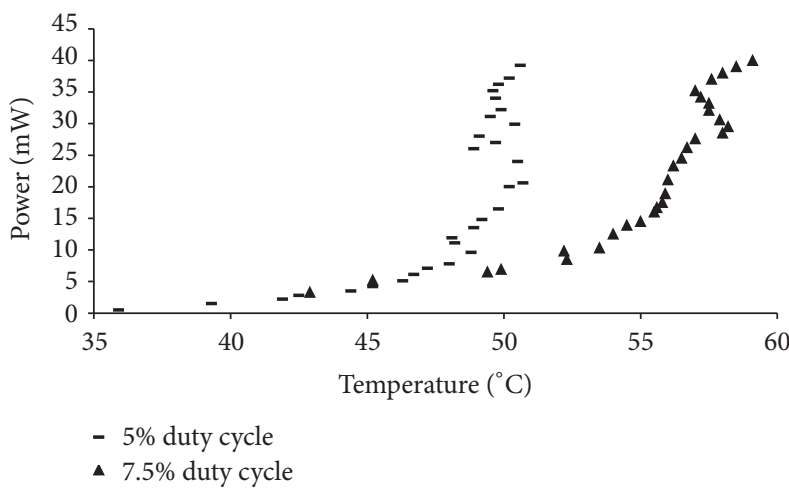

FIgURE 9: Transmission characteristics of $5 \%$ and $7.5 \%$ duty cycle.

Spot size $=10.69 \mathrm{~mm}$

$5 \%$ duty cycle

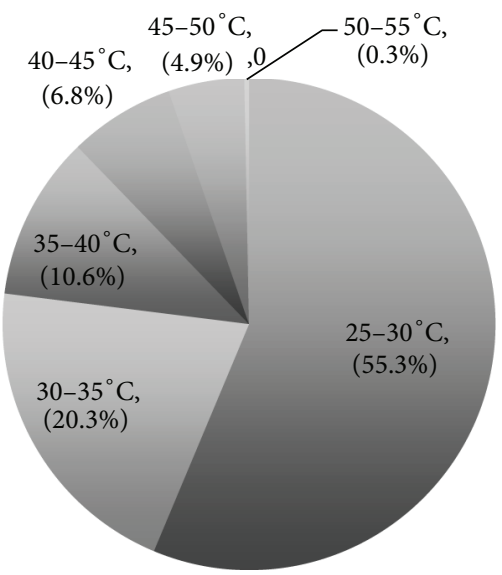

Spot size $=12.52 \mathrm{~mm}$

$7.5 \%$ duty cycle

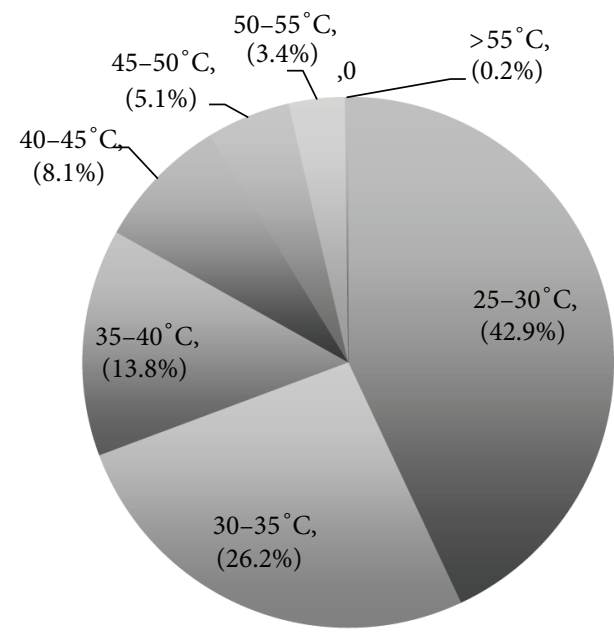

Figure 10: Pixel map of images at 5\% and 7.5\% duty cycle. 


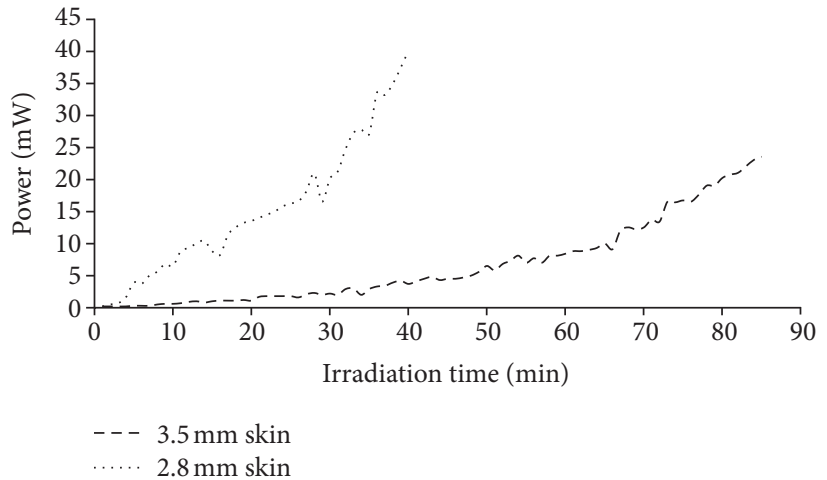

FIgURE 11: Temporal characteristics of $2.81 \mathrm{~mm}$ and $3.5 \mathrm{~mm}$ skin thicknesses.

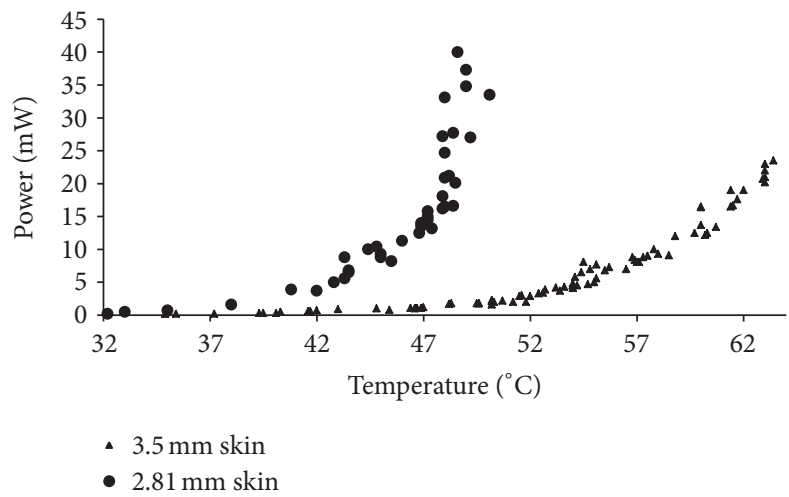

FIGURE 12: Transmission characteristics of $2.81 \mathrm{~mm}$ and $3.5 \mathrm{~mm}$ skin thicknesses.

the onset of radiation. As shown, the spot size and energy transferred increases with time and exposure. Samples were irradiated for a maximum time period up to 45-60 minutes at low input power of $83 \mathrm{~mW}$. The transmitted power and temperature signatures were measured every minute during irradiation exposure. An infrared video was also recorded for analysis purposes.

\section{Experimental Results}

Figure 3 represents the temperature distribution along $x$ axis and $y$-axis of the irradiated spot. From the figure, it is apparent that the surface temperature varies exponentially as a function of radial distance relative to the center of irradiation, where the temperature is the highest.

The optical power transmitted through skin varies with input pulse frequency. Figures 4 and 5 demonstrate that transmitted power increases exponentially with skin temperature, and higher frequency pulses serve to transmit more optical power than lower frequency pulses at equivalent temperature. The surface temperature of the skin was recorded at the center of irradiation and includes an area of $0.075 \mathrm{~mm}^{2}$.

Using infrared thermography, pixel maps (thermal images) of the irradiated spot were collected to study the temperature distribution around the irradiated spot for various pulse parameters. Figure 6 compares pixel map statistics of $10-\mathrm{kHz}$ and $100-\mathrm{kHz}$ pulsed trains and reveals a larger percentage of high temperature pixels for $100-\mathrm{kHz}$ samples.

The absorption characteristics of the irradiated skin were observed and approximated for the various pulse parameters. The absorption coefficient $(u)$ for the skin (epidermis) was estimated using Beer-Lambert law. This remains an approximation because the scattering parameters were not determined. Figure 7 shows the absorption coefficients of the irradiated skin for $1-\mathrm{kHz}$ and $10-\mathrm{kHz}$ pulsed trains at various sample temperatures. Clearly the absorption decreases as the sample temperature increases, and this is attributed to the decrease in skin water content during sample exposure. Altering the duty cycle of the pulse train was performed and it was shown to also vary the transmitted optical power. Figure 8 illustrates that a higher duty cycle pulsed wave (5\%) transmits more power than lower duty cycle wave (3\%) at equal time interval. However, Figure 9 shows that lower duty cycle pulse wave $(5 \%)$ transmits more optical power than higher duty cycle pulse wave (7.5\%) at equal temperature. The pixel map (thermal images) of irradiated spot taken at different duty cycles was also recorded and the statistics are provided in Figure 10.

Obviously, each skin sample is unique with reference to its thickness. As expected, it was observed that thinner skin samples transmitted more optical power at lower temperature and at a faster rate than thicker skin samples. Figures 11 

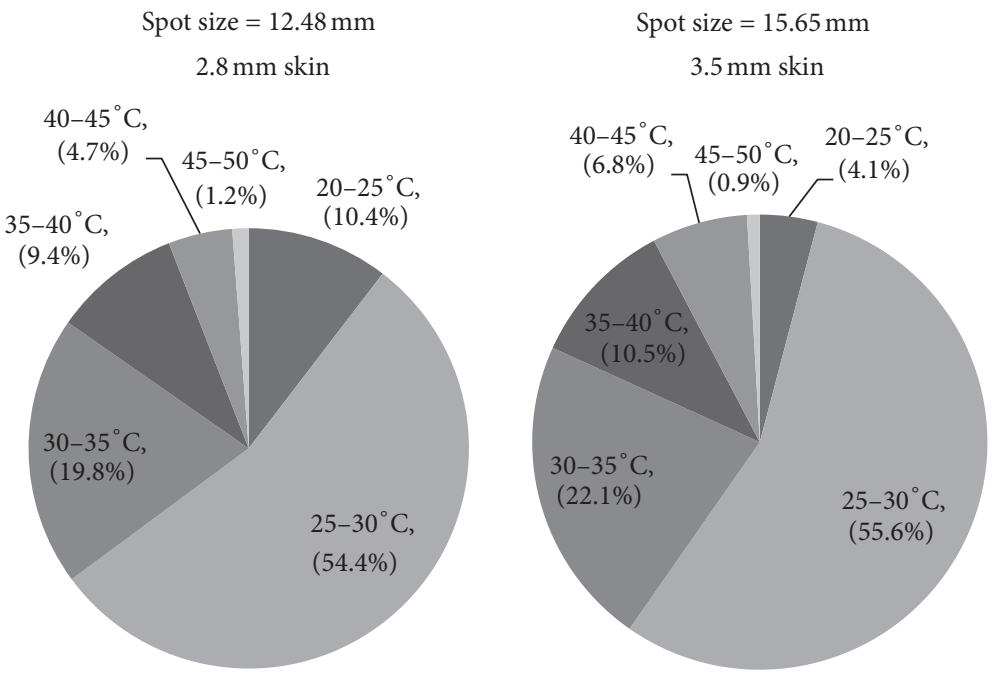

FIGURE 13: Pixel map comparison of images taken from samples with varying thickness.

and 12 represent the transmission characteristics of samples with various thicknesses at equal infrared exposure time and at equivalent temperature, respectively. Figure 13 represent the pixel mapping for samples with various thickness. The impact of skin thickness is obvious, and the transmission efficiencies are distinguishable. The energy density calculated at maximum transmission for $2.8 \mathrm{~mm}$ skin is $(12 \mathrm{~J}) / \mathrm{cm}^{2}$, and this falls within the energy range required for photonic biostimulation [8].

\section{Discussion}

When skin is exposed to collimated near-infrared light, its thermal states changes resulting in an increase in surface temperature at the onset of irradiation; however, the surrounding surface area is at a lower temperature resulting in a significant temperature gradient, which follows with noticeable heat transfer to the sublayers of skin. This loss of optical energy to the surrounding skin is attributed to absorption and dissipation processes, which is attributed to the nonuniformity skin layers. The uniform drop in temperature around the irradiated area, however, illustrates that associated transmission losses is uniform and nearly isotropic (near the irradiation spot, Figure 3) and likely occurs in the uppermost layer of skin. The results prove that optical power transmitted through skin increases with irradiation exposure and time (Figures 4 and 5), although this exponential rise varies with respect to frequency of pulsed beam. At higher frequencies, where there is a comparative decrease in the pulse width of the beam and interpulse duration, the thermal energy builds up more efficiently, thus increasing skin temperature, temperature gradient, and subsequent transmission. In order to transmit comparable power using a low frequency pulsed wave, the skin samples must reside at an initial higher temperature or have an increased exposure time. The pixel map collect by infrared thermography (Figure 6) highlights the influence of pulse frequency. The $100-\mathrm{kHz}$ wave has a higher percentage of pixels in the maximum $\left(50-55^{\circ} \mathrm{C}\right)$ and minimum $\left(25-30^{\circ} \mathrm{C}\right)$ temperature range. This signifies increased thermal build up and residual losses, whereas the $10-\mathrm{kHz}$ pulsed wave generates a higher percentage in the $30-50^{\circ} \mathrm{C}$ range, signifying greater dissipation to the surrounding skin and therefore provides reduced transmission efficiency.

Obviously, the transmission efficiency is not only dependent on the temperature, but also on the moisture content. As the sample temperature increases gradually, the moisture content continuously decreases (Figure 7). These transmission characteristics are comparable to water, whose absorption coefficient is also inversely proportional with temperature [9]. As skin samples contain up to $80 \%$ water [10], its absorption should also decrease with temperature and the results shown demonstrate the same. The reduction in the absorption coefficient is more prominent for higher frequencies, since higher frequencies provide increased buildup of thermal energy. Appropriately, transmission efficiency can be modeled empirically and controlled by carefully altering beam parameters.

Temporal characteristics shown in Figure 8 suggest that higher duty cycle pulsed wave (5\%) transmits more optical power in a shorter time duration because the higher duty cycle has a longer "ON state" in comparison to the lower duty cycle (3\%) pulsed wave. The longer "ON state" signifies that more input power is delivered, thus causing an increase in temperature and therefore an increase in transmission during the same time interval. Though the power transmitted increases at a faster rate, the temperature build up is not as obvious in the higher duty cycle pulsed wave for the same reason, that is, longer "ON state." Transmission characteristics in Figure 9 also show that lower duty cycle pulsed waves (5\%) transmit more optical power than higher duty cycle pulsed waves $(7.5 \%)$ at equal temperature. If careful selection of the duty cycle is ignored, it may result in overheating of skin and result in high thermal losses, as energy is not efficiently transferred through the skin. Figure 10, on the other hand shows that low-duty cycle pulsed wave (5\%) has 
a greater percentage of temperature in the $25-30^{\circ} \mathrm{C}$ range, signifying efficient transmission and reduced loss, while higher duty cycle pulsed wave $(7.5 \%)$ has greater percentage of temperature in the $30-55^{\circ} \mathrm{C}$, denoting that it experiences overheating, which in turn results in inefficient transmission of optical power.

The importance of skin thickness is also highlighted in this study. In Figure 11, it is evident that at equal exposure times, thick skin transmits more optical power than thinner skin. However, regardless of sample thickness, all samples can transmit equivalent power, albeit, at the expense of dosage. Figures 12 and 13 further illustrate this effect, that is, thinner skin requires less dosage and experiences reduced dissipation losses.

\section{Summary}

Optical studies were conducted to explore the influence of controllable infrared source parameters on optical transmission through skin and demonstrated a maximum efficiency of $86 \%$. All studies exhibited variations in transmission efficiency, proving that the percentage of power transmitted can be appropriate tuned. The frequency study demonstrated that higher frequencies transmit more optical power at a lower skin temperature, whereas the duty cycle study revealed the following: higher duty cycle can transmit greater power at reduced exposure times and lower duty cycle can transmit higher power at lower temperatures. The transmission characteristics of pigskin also reveal that optical power transmitted through skin increases exponentially with skin temperature, while the absorption characteristics show that the absorption coefficient decreases linearly with increasing temperature, which is similar to the absorption characteristic of water. Depending on the application, the pulsing parameters and initial sample temperature can be carefully selected to enhance biostimulation. In an application related to cell regeneration, for example, frequency modulation would be beneficial as it aids in transmitting optical power at lower temperature. On the other hand, where sample heating is more significant, the focus on duty cycle would be beneficial.

\section{References}

[1] J. Ashley Welch and J. C. Martin van Gemert, Optical-Thermal Response of Laser-Irradiated Tissue, Springer, New York, NY, USA, 2011.

[2] J. Michael Conlan, W. John Rapley, and M. Charles Cobb, "Biostimulation of wound healing by low-energy laser irradiation A review," Journal of Clinical Periodontology, vol. 23, no. 5, pp. 492-496, 1996.

[3] R. Marks and P. A. Payne, Bioengineering and the Skin, Kluwer Academic Publishers, Hingham, Mass, USA, 1981.

[4] D. Yudovsky and L. Pilon, "Rapid and accurate estimation of blood saturation, melanin content, and epidermis thickness from spectral diffuse reflectance," Applied Optics, vol. 49, no. 10, pp. 1707-1719, 2010.

[5] A. G. Maiya, P. Kumar, and S. Nayak, "Photo-stimulatory effect of low energy helium-neon laser irradiation on excisional diabetic wound healing dynamics in wistar rats," Indian Journal of Dermatology, vol. 54, no. 4, pp. 323-329, 2009.
[6] R. D. Lins, E. M. Dantas, K. C. Lucena, M. H. Catão, A F. Granville-Garcia, and L. G. Carvalho Neto, "Biostimulation effects of low-power laser in the repair process," Anais Brasileiros de Dermatologia, vol. 85, no. 6, pp. 849-855, 2010.

[7] Z. Zhang and N. A. Monteiro-Riviere, "Comparison of integrins in human skin, pig skin, and perfused skin: an in vitro skin toxicology model," Journal of Applied Toxicology, vol. 17, no. 4, pp. 247-253, 1997.

[8] O. T. Tan, T. J. Stafford, S. Murray, and A. K. Kurban, "Histologic comparison of the pulsed dye laser and copper vapor laser effects on pig skin," Lasers in Surgery and Medicine, vol. 10, no. 6, pp. 551-558, 1990.

[9] B. I. Lange, T. Brendel, and G. Hüttmann, "Temperature dependence of light absorption in water at holmium and thulium laser wavelengths," Applied Optics, vol. 41, no. 27, pp. 5797-5803, 2002.

[10] E. J. Suh, Y. A. Woo, and H. J. Kim, "Determination of water content in skin by using a FT near infrared spectrometer," Archives of Pharmacal Research, vol. 28, no. 4, pp. 458-462, 2005. 

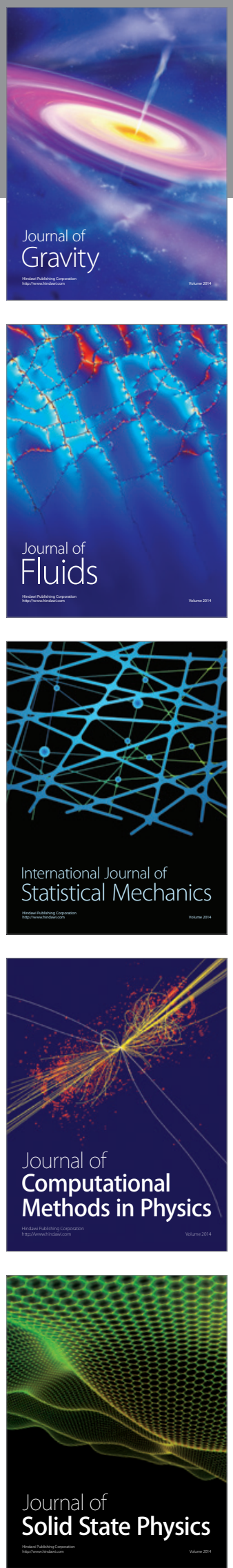

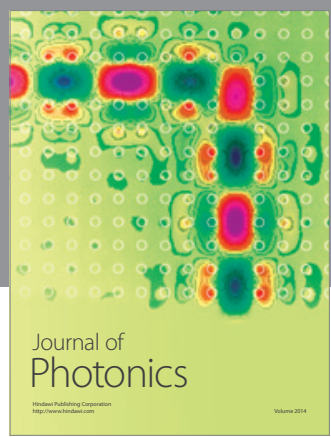

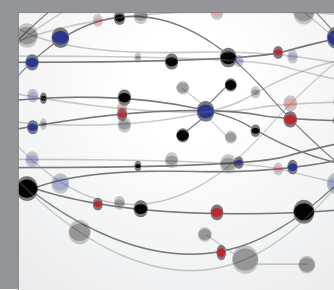

The Scientific World Journal

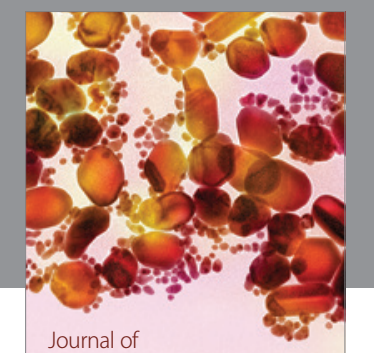

Soft Matter
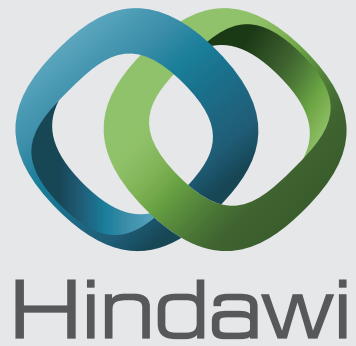

Submit your manuscripts at

http://www.hindawi.com
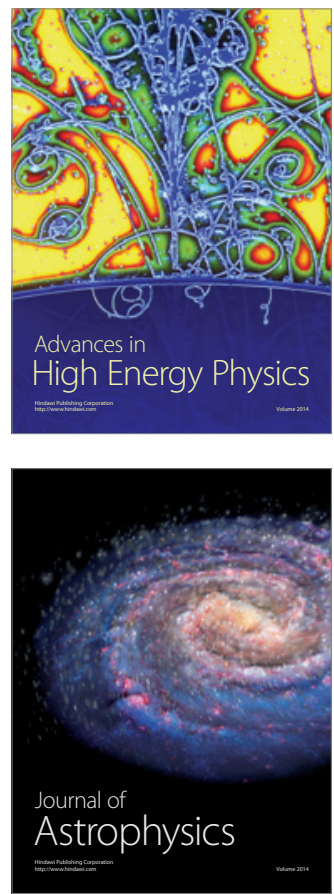
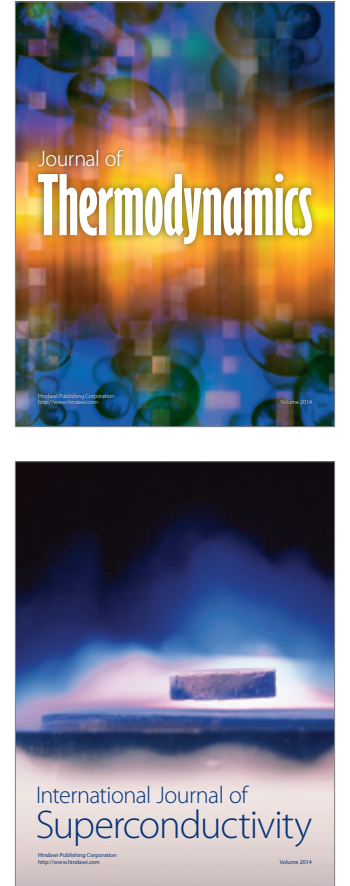
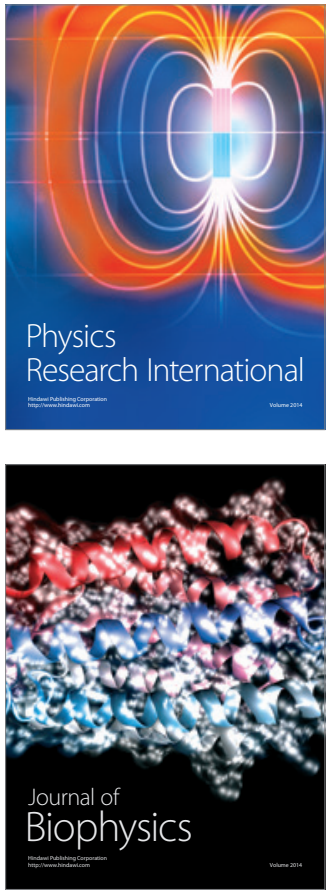
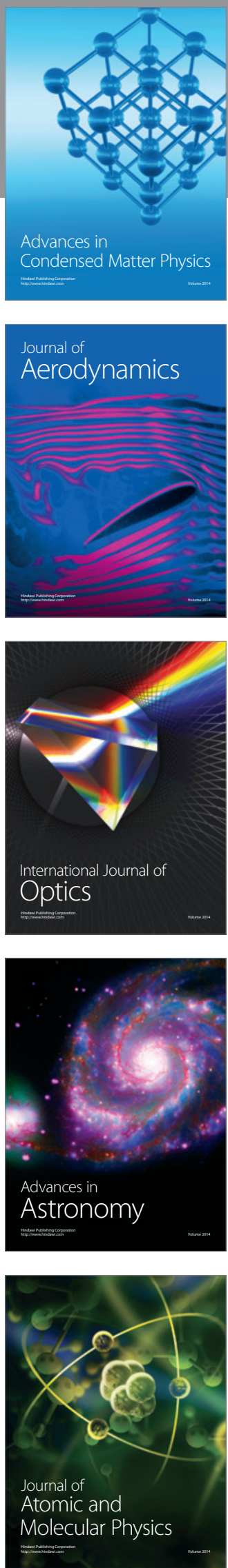